\title{
On values of a polynomial at arithmetic progressions with equal products
}

\author{
by
}

\author{
N. Saradha (Bombay), T. N. Shorey (Bombay) \\ and R. TiJdeman (Leiden)
}

1. Introduction. Let $f(X)$ be a monic polynomial of degree $\nu>0$ with rational coefficients. Let $d_{1}, d_{2}, l, m$ with $l<m$ and $\operatorname{gcd}(l, m)=1$ be given positive integers. In this paper, we consider the equation

$$
f(x) f\left(x+d_{1}\right) \ldots f\left(x+(l k-1) d_{1}\right)=f(y) f\left(y+d_{2}\right) \ldots f\left(y+(m k-1) d_{2}\right)
$$

in integers $x, y$ and $k \geq 2$ such that

$$
f\left(x+j d_{1}\right) \neq 0 \quad \text { for } 0 \leq j \leq l k-1 .
$$

We refer to [3] and [4] for an account of results on equation (1) with $f(X)=$ $X$. It was shown in [3] that for positive integers $x, y$ and $k \geq 2$, equation (1) with $f(X)=X$ implies that $\max (x, y, k) \leq C_{1}$ where $C_{1}$ is an effectively computable number depending only on $d_{1}, d_{2}, m$ unless

$$
l=1, \quad m=k=2, \quad d_{1}=2 d_{2}^{2}, \quad x=y^{2}+3 d_{2} y .
$$

When $f$ is a power of an irreducible polynomial, it was shown in [1] that equation (1) with $l=d_{1}=d_{2}=1$ and (2) implies that $\max (|x|,|y|, k) \leq C_{2}$ where $C_{2}$ is an effectively computable number depending only on $m$ and $f$. In this paper, we extend these results as follows.

Theorem. (a) Equation (1) with (2) implies that $k$ is bounded by an effectively computable number depending only on $d_{1}, d_{2}, m$ and $f$.

(b) Let $f$ be a power of an irreducible polynomial. There exists an effectively computable number $C_{3}$ depending only on $d_{1}, d_{2}, m$ and $f$ such that equation (1) with (2) implies that

$$
\max (|x|,|y|, k) \leq C_{3}
$$

unless

$$
\begin{gathered}
l=1, \quad m=k=2, \quad d_{1}=2 d_{2}^{2}, \\
f(X)=(X+r)^{\nu} \quad \text { with } r \in \mathbb{Z}, x+r=(y+r)\left(y+r+3 d_{2}\right) .
\end{gathered}
$$


It is clear that condition (2) is necessary. We observe that equation (1) is, in fact, satisfied in the cases given by (5). For irreducible $f$, we apply Theorem (b) to $f^{2}$ for deriving that if $x, y$ and $k \geq 2$ are integers satisfying (2) and

$\left|f(x) f\left(x+d_{1}\right) \ldots f\left(x+(l k-1) d_{1}\right)\right|=\left|f(y) f\left(y+d_{2}\right) \ldots f\left(y+(m k-1) d_{2}\right)\right|$

then $\max (|x|,|y|, k)$ is bounded by an effectively computable number depending only on $d_{1}, d_{2}, m$ and $f$ unless (5) holds. In particular, we observe that if $x, y$ and $k \geq 2$ are integers satisfying $x+j d_{1} \neq 0$ for $0 \leq j \leq l k-1$ and

$$
x\left(x+d_{1}\right) \ldots\left(x+(l k-1) d_{1}\right)= \pm y\left(y+d_{2}\right) \ldots\left(y+(m k-1) d_{2}\right)
$$

then $\max (|x|,|y|, k)$ is bounded by an effectively computable number depending only on $d_{1}, d_{2}$ and $m$, unless (3) holds.

2. Notation. Let $\left\{\alpha_{1}, \alpha_{2}, \ldots, \alpha_{\nu}\right\}$ be the roots of $f$ and we assume without loss of generality that $\left|\alpha_{1}\right| \geq\left|\alpha_{2}\right| \geq \ldots \geq\left|\alpha_{\nu}\right|$. Let $a_{0}$ be the absolute value of the product of the denominators of the coefficients of $f$. We observe that $a_{0} \alpha_{1}, \ldots, a_{0} \alpha_{\nu}$ are algebraic integers. We define the coefficients $A_{0}, A_{1}, \ldots$ and $B_{0}, B_{1}, \ldots$ by

$X^{-l} \prod_{j=0}^{l k-1}\left(f\left(X+j d_{1}\right)\right)^{1 /(\nu k)}=\prod_{i=1}^{\nu} \prod_{j=0}^{l k-1}\left(1+\frac{j d_{1}-\alpha_{i}}{X}\right)^{1 /(\nu k)}=\sum_{n=0}^{\infty} A_{n} d_{1}^{n} X^{-n}$

and

$$
\begin{aligned}
Y^{-m} \prod_{j=0}^{m k-1}\left(f\left(Y+j d_{2}\right)\right)^{1 /(\nu k)} & =\prod_{i=1}^{\nu} \prod_{j=0}^{m k-1}\left(1+\frac{j d_{2}-\alpha_{i}}{Y}\right)^{1 /(\nu k)} \\
& =\sum_{n=0}^{\infty} B_{n} d_{2}^{n} Y^{-n}
\end{aligned}
$$

We observe that for $n \geq 1, A_{n}$ and $B_{n}$ are rational numbers and that $A_{0}=B_{0}=1$. We put

$$
\chi_{n}=\left(\left(a_{0} \nu k\right) n !\right)^{n} \quad \text { for } n=0,1,2, \ldots
$$

Further, we write

$$
\begin{aligned}
& F(X)=X^{l}+A_{1} d_{1} X^{l-1}+\ldots+A_{l} d_{1}^{l}, \\
& G(Y)=Y^{m}+B_{1} d_{2} Y^{m-1}+\ldots+B_{m} d_{2}^{m}
\end{aligned}
$$

and

$$
L(X, Y)=F(X)-G(Y) .
$$

We notice that $F(X)$ and $G(Y)$ are the polynomial parts of the $\nu k$ th root of left and right hand sides of equation (1), respectively, with $x$ and $y$ re- 
placed by $X$ and $Y$. For a rational number $\beta$, we write $d(\beta)$ for the least positive integer such that $d(\beta) \beta$ is a rational integer. We denote by $c_{1}, c_{2}, \ldots$ effectively computable positive numbers depending on $d_{1}, d_{2}, m$ and $f$.

3. $k$ is bounded. In this section, we shall show that equation (1) with (2) implies that $k \leq c_{1}$. The proof is similar to that of Theorem 2 of [1]. Therefore, we mention only the main steps of the proof and the readers are referred to [1] for details. We assume that equation (1) with (2) is satisfied. Then we observe that

$$
|x|^{l} \leq c_{2}\left(|y|+m k d_{2}\right)^{m}, \quad|y|^{m} \leq c_{3}\left(|x|+l k d_{1}\right)^{l} .
$$

For $n \geq 0, A_{n}$ and $B_{n}$ are polynomials in $k$ of degrees not exceeding $n$ satisfying

$$
\left|A_{n}\right| d_{1}^{n} \leq 2^{n+l}\left(l k d_{1}+\left|\alpha_{1}\right|\right)^{n}, \quad\left|B_{n}\right| d_{2}^{n} \leq 2^{n+m}\left(m k d_{2}+\left|\alpha_{1}\right|\right)^{n}
$$

and

Further, we obtain

$$
d\left(A_{n} d_{1}^{n}\right)\left|\chi_{n}, \quad d\left(B_{n} d_{2}^{n}\right)\right| \chi_{n} .
$$

$$
\log (|y|+2) \geq c_{4} k
$$

For the proof of (7), we take prime $p$ of Lemma 4 of [1] exceeding $a_{0} d_{1} d_{2}$ in place of $a_{0}$.

We assume from now onward that $|y|>c_{5}$ with $c_{5}$ sufficiently large, otherwise (4) follows from (7) and (6). By taking $\nu k$ th root on both the sides of equation (1), we have

$$
x^{l}\left(1+\frac{A_{1} d_{1}}{x}+\frac{A_{2} d_{1}^{2}}{x^{2}}+\ldots\right)=y^{m}\left(1+\frac{B_{1} d_{2}}{y}+\frac{B_{2} d_{2}^{2}}{y^{2}}+\ldots\right) .
$$

This implies that

$$
F(x)=G(y)
$$

Further, we show that

$$
A_{l+1}=\ldots=A_{2 l-1}=0 \quad \text { or } \quad B_{m+1}=\ldots=B_{2 m-1}=0 .
$$

We prove (9) by contradiction. If not, there exist integers $I$ and $J$ with $1 \leq I<l$ and $1 \leq J<m$ such that

$$
A_{l+1}=\ldots=A_{l+I-1}=0, \quad A_{l+I} \neq 0
$$

and

$$
B_{m+1}=\ldots=B_{m+J-1}=0, \quad B_{m+J} \neq 0 .
$$

Then we derive that

$$
\frac{A_{l+I} d_{1}^{l+I}}{x^{I}}+\ldots=\frac{B_{m+J} d_{2}^{m+J}}{y^{J}}+\ldots,
$$


which implies that $m I=l J$. This is not possible since $\operatorname{gcd}(l, m)=1$ and $J<m$. Further, we derive from (8) and (9) that

$$
A_{l+1}=\ldots=A_{2 l-1}=0, \quad B_{m+1}=\ldots=B_{2 m-1}=0
$$

and

$$
B_{2 m} d_{2}^{2 m}=A_{2 l} d_{1}^{2 l} .
$$

Finally, we apply the proof of $\S 4$ of [1] for deriving from the above relations that $k \leq c_{1}$. This completes the proof of Theorem (a).

4. Proof of Theorem (b). We assume that equation (1) with (2) is satisfied. Then, by Theorem (a), we restrict ourselves to $k \leq c_{1}$. Let $k$ be fixed. By (6), we may assume that $|x|>c_{5}$ and $|y|>c_{5}$ with $c_{5}$ sufficiently large. Then the relation (8) is valid. Let $f=g_{1}^{b}$, where $g_{1}$ is irreducible and $b$ is a positive integer. Then $g_{1}$ has rational coefficients and its leading coefficient is \pm 1 . By putting $f=g_{1}^{b}$ in (1), we have

$$
\begin{aligned}
\left(g_{1}(x) g_{1}\left(x+d_{1}\right) \ldots g_{1}(x+\right. & \left.\left.(l k-1) d_{1}\right)\right)^{b} \\
& =\left(g_{1}(y) g_{1}\left(y+d_{2}\right) \ldots g_{1}\left(y+(m k-1) d_{2}\right)\right)^{b} .
\end{aligned}
$$

Taking the $b$ th root on either side, we see that

$$
\begin{aligned}
g_{1}(x) g_{1}\left(x+d_{1}\right) \ldots g_{1}(x+ & \left.(l k-1) d_{1}\right) \\
& = \pm g_{1}(y) g_{1}\left(y+d_{2}\right) \ldots g_{1}\left(y+(m k-1) d_{2}\right) .
\end{aligned}
$$

Now, we set $g_{1}(x)=g(x)$ if $g_{1}$ is monic and $g_{1}(x)=-g(x)$ if $g_{1}$ has leading coefficient -1 so that $g$ is a monic irreducible polynomial with rational coefficients. Then the latter equation is valid with $g_{1}$ replaced by $g$. Thus we assume that either $f=g$ or $f=g^{2}$ in Theorem (b). Put $\delta=1$ if $f=g$ and $\delta=2$ if $f=g^{2}$. Let $\mu$ be the degree of $g$. Thus $\mu=\nu / \delta$. Let $\beta_{1}, \ldots, \beta_{\mu}$ be the roots of $g, K=\mathbb{Q}\left(\beta_{1}, \ldots, \beta_{\mu}\right)$ and we write $a$ for the coefficient of $X^{\mu-1}$ in $g(X)$. Further, let $\sigma_{1}, \ldots, \sigma_{\mu}$ be all the automorphisms of $K$ and we write $\sigma_{q}(\beta)=\beta^{(q)}$ for $\beta \in K$ and $1 \leq q \leq \mu$. We set

$$
\begin{aligned}
H(X, Y) & =\left(g(X) \ldots g\left(X+(l k-1) d_{1}\right)\right)^{\delta}-\left(g(Y) \ldots g\left(Y+(m k-1) d_{2}\right)\right)^{\delta}, \\
T & =\left\{\beta_{i}-J d_{1} \mid 1 \leq i \leq \mu, 0 \leq J<l k\right\}
\end{aligned}
$$

and

$$
U=\left\{\beta_{i}-J d_{2} \mid 1 \leq i \leq \mu, 0 \leq J<m k\right\} .
$$

Since $g$ is irreducible, we observe that $|T|=l k \mu$ and $|U|=m k \mu$. For $t=$ $\beta_{i}-J d_{1} \in T$, we write $\bar{t}=J d_{1}$. Similarly, for $u=\beta_{i}-J d_{2} \in U$, we write $\bar{u}=J d_{2}$.

Let $R(Y)$ be the resultant of $H(X, Y)$ and $L(X, Y)$ with respect to $X$. Then we observe from equations (1) and (8) that $R(y)=0$, which implies that $R(Y) \equiv 0$ if $c_{5}$ is sufficiently large. By a result of Ehrenfeucht (see 
[2, p. 2]), $L(X, Y)$ is irreducible over the field of complex numbers since $\operatorname{gcd}(l, m)=1$. Therefore, $L(X, Y)$ divides $H(X, Y)$, which implies that

$$
L(X, Y) \mid\left(g(X) \ldots g\left(X+(l k-1) d_{1}\right) \pm g(Y) \ldots g\left(Y+(m k-1) d_{2}\right)\right) .
$$

Thus

$$
F(X)-G(u) \mid g(X) \ldots g\left(X+(l k-1) d_{1}\right) \quad \text { for } u \in U
$$

and

$$
G(Y)-F(t) \mid g(Y) \ldots g\left(Y+(m k-1) d_{2}\right) \quad \text { for } t \in T
$$

over $K$.

Let $v_{1}^{\prime}, \ldots, v_{s^{\prime}}^{\prime}$ be the distinct values in $\{F(t) \mid t \in T\}$ and $v_{1}^{\prime \prime}, \ldots, v_{s^{\prime \prime}}^{\prime \prime}$ be the distinct values in $\{G(u) \mid u \in U\}$. Each $v_{i}^{\prime}$ is assumed by $F$ at most $l$ times. Therefore, $l k \mu \leq l s^{\prime}$, which implies that $k \mu \leq s^{\prime}$. Further, $G(y)-v_{i}^{\prime}$ with $1 \leq i \leq s^{\prime}$ are relatively coprime polynomials. Therefore, the product of these polynomials divides $g(Y) \ldots g\left(Y+(m k-1) d_{2}\right)$. Thus $m s^{\prime} \leq m k \mu$, which implies that $s^{\prime} \leq k \mu$. Hence, we conclude that $s^{\prime}=k \mu$ and each $v_{i}^{\prime}$ is assumed by $F$ exactly $l$ times in $T$. By a similar argument, we have $s^{\prime \prime}=k \mu$ and each $v_{i}^{\prime \prime}$ is assumed by $G$ exactly $m$ times in $U$. Thus, $s^{\prime}=s^{\prime \prime}=k \mu=: s$. Further, we have

$$
g(X) \ldots g\left(X+(l k-1) d_{1}\right)=\prod_{i=1}^{s}\left(F(X)-v_{i}^{\prime \prime}\right)
$$

and

$$
g(Y) \ldots g\left(Y+(m k-1) d_{2}\right)=\prod_{i=1}^{s}\left(G(Y)-v_{i}^{\prime}\right) .
$$

We write

$$
\prod_{i=1}^{s}\left(F(X)-v_{i}^{\prime \prime}\right)=(F(X))^{s}+A_{1}^{\prime}(F(X))^{s-1}+\ldots+A_{s}^{\prime}
$$

and

$$
\prod_{i=1}^{s}\left(G(Y)-v_{i}^{\prime}\right)=(G(Y))^{s}+B_{1}^{\prime}(G(Y))^{s-1}+\ldots+B_{s}^{\prime} .
$$

As $g(x) g\left(x+d_{1}\right) \ldots g\left(x+(l k-1) d_{1}\right)= \pm g(y) g\left(y+d_{2}\right) \ldots g\left(y+(m k-1) d_{2}\right)$, by (8) we have either

$$
\left(A_{1}^{\prime}-B_{1}^{\prime}\right)(F(x))^{s-1}+\ldots+\left(A_{s}^{\prime}-B_{s}^{\prime}\right)=0
$$

or

$$
2(F(x))^{s}+\left(A_{1}^{\prime}+B_{1}^{\prime}\right)(F(x))^{s-1}+\ldots+\left(A_{s}^{\prime}+B_{s}^{\prime}\right)=0 .
$$

If $c_{5}$ is sufficiently large, the latter possibility is excluded and the former possibility implies that $A_{1}^{\prime}=B_{1}^{\prime}, \ldots, A_{s}^{\prime}=B_{s}^{\prime}$. Consequently, we conclude 
that

$$
\left\{v_{1}^{\prime}, \ldots, v_{s}^{\prime}\right\}=\left\{v_{1}^{\prime \prime}, \ldots, v_{s}^{\prime \prime}\right\} .
$$

By rearrangement, if necessary, we may assume without loss of generality that $v_{i}^{\prime}=v_{i}^{\prime \prime}=: v_{i}$ for $1 \leq i \leq s$ and we write $S=\left\{v_{1}, \ldots, v_{s}\right\}$. Then we have

$$
F(X)-v_{i}=\left(X-t_{i, 1}\right) \ldots\left(X-t_{i, l}\right) \quad \text { for } 1 \leq i \leq s
$$

and

$$
G(Y)-v_{i}=\left(Y-u_{i, 1}\right) \ldots\left(Y-u_{i, m}\right) \quad \text { for } 1 \leq i \leq s,
$$

where $t_{i, p}=\gamma_{i, p}-\bar{t}_{i, p}$ for $1 \leq p \leq l$ and $u_{i, h}=\beta_{i, h}-\bar{u}_{i, h}$ for $1 \leq h \leq m$. Here $\gamma_{i, p}$ and $\beta_{i, h}$ belong to $\left\{\beta_{1}, \ldots, \beta_{\mu}\right\}$.

We now fix $i$ with $1 \leq i \leq s$ and let $r$ be the number of automorphisms of $K$ which fix $v_{i}$. By re-arranging $\sigma_{1}, \ldots, \sigma_{\mu}$, there is no loss of generality in assuming that $\sigma_{q}\left(v_{i}\right)=v_{i}^{(q)}=v_{i}$ for $1 \leq q \leq r$. The sets $\left\{\sigma_{q}\left(t_{i, p}\right) \mid\right.$ $1 \leq q \leq r\}$ for $1 \leq p \leq l$ are either disjoint or identical. Consequently, by considering the images under $\sigma_{q}$ with $1 \leq q \leq r$ on both sides of (10), we observe that the number of times $\bar{t}_{i, p}$ with $1 \leq p \leq l$ occurs in $\left\{\bar{t}_{i, 1}, \ldots, \bar{t}_{i, l}\right\}$ is a multiple of $r$. Consequently, we derive that $l$ is a multiple of $r$. Similarly, by considering (11) and arguing as above, we derive that $m$ is also a multiple of $r$. Since $\operatorname{gcd}(l, m)=1$, we have $r=1$. In other words, every element of $S$ has $\mu$ distinct conjugates. Therefore, the maximal number of elements of $S$ such that no two of them are conjugates is precisely $k$. By re-arranging elements of $S$, we may assume that $v_{1}, \ldots, v_{k}$ are such that no two of them are conjugates. Then we derive from (10) and (11) that $\bar{t}_{i, p}$ with $1 \leq i \leq$ $k, 1 \leq p \leq l$ are pairwise distinct elements of the set $\left\{J d_{1} \mid 0 \leq J<l k\right\}$ and $\bar{u}_{i, h}$ with $1 \leq i \leq k, 1 \leq h \leq m$ are pairwise distinct elements of the set $\left\{J d_{2} \mid 0 \leq J<m k\right\}$. By subtracting (10) with $X=x$ from (11) with $Y=y$ and taking norms over $K$, we derive that

$$
g\left(x+\bar{t}_{i, 1}\right) \ldots g\left(x+\bar{t}_{i, l}\right)=g\left(y+\bar{u}_{i, 1}\right) \ldots g\left(y+\bar{u}_{i, m}\right) \quad \text { for } 1 \leq i \leq k .
$$

Let $1 \leq i, j \leq k$ with $i \neq j$. This is possible since $k \geq 2$. We derive from (12) that

$$
\frac{g\left(x+\bar{t}_{i, 1}\right) \ldots g\left(x+\bar{t}_{i, l}\right)}{g\left(x+\bar{t}_{j, 1}\right) \ldots g\left(x+\bar{t}_{j, l}\right)}=\frac{g\left(y+\bar{u}_{i, 1}\right) \ldots g\left(y+\bar{u}_{i, m}\right)}{g\left(y+\bar{u}_{j, 1}\right) \ldots g\left(y+\bar{u}_{j, m}\right)} .
$$

Taking logarithms on both sides, we get

$$
\frac{V_{1}}{x}+\frac{V_{2}}{x^{2}}+\ldots=\frac{W_{1}}{y}+\frac{W_{2}}{y^{2}}+\ldots
$$

for certain numbers $V_{e}, W_{e}$, satisfying $\max \left(\left|V_{e}\right|,\left|W_{e}\right|\right) \leq c_{6}^{e}$ for $e \geq 1$. In 
fact, we have

$$
W_{e}=\frac{(-1)^{e-1}}{e} \sum_{h=1}^{m} \sum_{q=1}^{\mu}\left\{\left(\bar{u}_{i, h}-\beta_{q}\right)^{e}-\left(\bar{u}_{j, h}-\beta_{q}\right)^{e}\right\} .
$$

Now, we shall derive that

$$
V_{1}=\ldots=V_{l-1}=0, \quad W_{1}=\ldots=W_{m-1}=0 .
$$

We prove (13) by contradiction like we proved (9). Suppose $I$ and $J$ are integers with $1 \leq I<l, 1 \leq J<m$ such that $V_{1}=\ldots=V_{I-1}=0, V_{I} \neq$ $0, W_{1}, \ldots, W_{J-1}=0, W_{J} \neq 0$. Then

$$
\frac{V_{I}}{x^{I}}+\ldots=\frac{W_{J}}{y^{J}}+\ldots,
$$

which implies that $m I=l J$. Since $\operatorname{gcd}(l, m)=1$, this implies $l$ divides $I$ and $m$ divides $J$, whence (13) follows.

Now, by induction on $e$, it follows from (13) that

$$
W_{e}^{\prime}=\frac{(-1)^{e-1}}{e} \sum_{h=1}^{m}\left(\left(\bar{u}_{i, h}\right)^{e}-\left(\bar{u}_{j, h}\right)^{e}\right)
$$

satisfies $W_{1}^{\prime}=\ldots=W_{m-1}^{\prime}=0$. This implies that

$$
\log \frac{\prod_{h=1}^{m}\left(1+\bar{u}_{i, h} / y\right)}{\prod_{h=1}^{m}\left(1+\bar{u}_{j, h} / y\right)}=\frac{W_{m}^{\prime}}{y^{m}}+\ldots
$$

Thus

$$
\prod_{h=1}^{m}\left(y+\bar{u}_{i, h}\right)=\prod_{h=1}^{m}\left(y+\bar{u}_{j, h}\right)+W_{m}^{\prime}+O(1 / y) .
$$

By taking $y$ sufficiently large and writing $E_{i, j}$ for $W_{m}^{\prime}$, we get the polynomial relation

$$
\prod_{h=1}^{m}\left(Y+\bar{u}_{i, h}\right)=\prod_{h=1}^{m}\left(Y+\bar{u}_{j, h}\right)+E_{i, j} \quad \text { for } 1 \leq i, j \leq k, i \neq j
$$

for some number $E_{i, j}$. We observe that $E_{i, j} \neq 0$ for $1 \leq i, j \leq k$ and $i \neq j$.

We put

$$
g_{2}(Y)=\prod_{h=1}^{m}\left(Y+\bar{u}_{1, h}\right)
$$

By (14), we have

$$
g_{2}(Y)=\prod_{h=1}^{m}\left(Y+\bar{u}_{j, h}\right)+E_{j} \quad \text { for } 2 \leq j \leq k \text { with } E_{j}=E_{1, j} .
$$

We observe from (15) and (14) that $E_{j}$ for $2 \leq j \leq k$ are pairwise distinct non-zero numbers. Further, we see from (15) that every number $0=: E_{1}$, 
$E_{2}, \ldots, E_{k}$ is assumed by the polynomial $g_{2}$ at $m$ distinct integers from $\left\{-J d_{2} \mid 0 \leq J \leq m k-1\right\}$. Now, we may follow an argument of the proof of Theorem 2 of [3] to conclude that

$$
\max (|x|,|y|) \leq c_{7} \quad \text { unless } m=2 .
$$

This argument depends on Rolle's theorem. Here we give a proof of the preceding assertion without using Rolle's theorem.

As already observed, the elements of the sets $\bar{U}_{i}=\left\{\bar{u}_{i, 1}, \ldots, \bar{u}_{i, m}\right\}$ for $1 \leq i \leq k$ are distinct and $\bar{U}_{i} \cap \bar{U}_{j}=\emptyset$ for $i \neq j, 1 \leq i, j \leq k$. Then

$$
\sum_{i=1}^{k} \sum_{h=1}^{m} \bar{u}_{i, h}=\sum_{J=0}^{m k-1} J d_{2}=m k(m k-1) d_{2} / 2 .
$$

Further, by equating the coefficients of $Y^{m-1}$ on both sides of (14), we obtain

$$
\sum_{h=1}^{m} \bar{u}_{i, h}=\sum_{h=1}^{m} \bar{u}_{j, h} \quad \text { for } 1 \leq i, j \leq k .
$$

Consequently, we have

$$
\sum_{h=1}^{m} \bar{u}_{i, h}=m(m k-1) d_{2} / 2 \quad \text { for } 1 \leq i \leq k .
$$

We assume without loss of generality that

$$
\bar{u}_{i, 1}<\bar{u}_{i, 2}<\ldots<\bar{u}_{i, m} \quad \text { for } 1 \leq i \leq k
$$

and

$$
0=\bar{u}_{1,1}<\bar{u}_{2,1}<\ldots<\bar{u}_{k, 1} .
$$

We show by induction on $i$ that

$$
\bar{u}_{i, 1}=(i-1) d_{2} \quad \text { for } 1 \leq i \leq k .
$$

We observe that (20) with $i=1$ is true by (19). We assume that (20) is valid for $1 \leq i \leq i_{0}$ with $i_{0} \leq k-1$. If $i_{0} d_{2} \in \bar{U}_{i_{1}}$ with $1 \leq i_{1} \leq i_{0}$, we consider (14) with $i=i_{1}, j=i_{0}+1$ and we put $Y=-\left(i_{1}-1\right) d_{2},-i_{0} d_{2}$ to get a contradiction. Then (20) with $i=i_{0}+1$ follows from (18) and (19).

Next, we show by induction on $h$ that

$$
\bar{u}_{k, h}=(k+h-2) d_{2} \quad \text { for } 1 \leq h \leq m .
$$

If $h=1$, we observe that (21) is (20) with $i=k$. We suppose that $\bar{u}_{k, h}=$ $(k+h-2) d_{2}$ for $1 \leq h \leq h_{0}$ with $h_{0} \leq m-1$. If $\left(k+h_{0}-1\right) d_{2} \in \bar{U}_{i_{2}}$ with $1 \leq i_{2} \leq k-1$, we consider (14) with $i=i_{2}, j=k$ and we put $Y=-\left(i_{2}-1\right) d_{2},-\left(k+h_{0}-1\right) d_{2}$ to find that

$$
\begin{array}{r}
\left(k-i_{2}\right)\left(k-i_{2}+1\right) \ldots\left(k-i_{2}+h_{0}-1\right)\left(\bar{u}_{k, h_{0}+1}-\left(i_{2}-1\right) d_{2}\right) \ldots \\
\ldots\left(\bar{u}_{k, m}-\left(i_{2}-1\right) d_{2}\right)
\end{array}
$$




$$
\begin{gathered}
=(-1)^{h_{0}} h_{0}\left(h_{0}-1\right) \ldots 1\left(\bar{u}_{k, h_{0}+1}-\left(k+h_{0}-1\right) d_{2}\right) \ldots \\
\ldots\left(\bar{u}_{k, m}-\left(k+h_{0}-1\right) d_{2}\right) .
\end{gathered}
$$

This is not possible since $\left(k-i_{2}\right) \ldots\left(k-i_{2}+h_{0}-1\right) \geq h_{0}$ ! and (18). Hence (21) with $h=h_{0}+1$ follows. This completes the proof of (21). Then

$$
\sum_{h=1}^{m} \bar{u}_{k, h}=\left(m k+\frac{1}{2} m(m-3)\right) d_{2},
$$

which, together with (17), implies that $k=1$ whenever $m \geq 3$. This completes the proof of (16) without using Rolle's theorem.

Next we turn to the case $m=2$. Then $l=1$. Let $1 \leq i<j \leq k$. It follows from (13) that the corresponding $W_{1}$ satisfies $W_{1}=0$. Extending the argument used for proving (13) we see that $V_{1}=W_{2}$. By definition $V_{1}=$ $\mu\left(\bar{t}_{i, 1}-\bar{t}_{j, 1}\right)$. Further, by $W_{1}=0$, we have $E_{i, j}=W_{2}^{\prime}=W_{2}$. Consequently, $E_{i, j}=\mu\left(\bar{t}_{i, 1}-\bar{t}_{j, 1}\right)$. Hence and from (14), (20) and (17) we derive

$$
\begin{aligned}
\left(Y+(i-1) d_{2}\right) & \left(Y+(2 k-i) d_{2}\right) \\
= & \left(Y+(j-1) d_{2}\right)\left(Y+(2 k-j) d_{2}\right)+\mu\left(\bar{t}_{i, 1}-\bar{t}_{j, 1}\right) .
\end{aligned}
$$

Since $z(2 k-1-z)$ is an increasing function for $0 \leq z \leq k-1$, it follows that $\bar{t}_{i, 1}<\bar{t}_{j, 1}$ for $i<j$. Thus

$$
\bar{t}_{i, 1}=(i-1) d_{1} \quad \text { for } 1 \leq i \leq k .
$$

Suppose first $k \geq 3$. From (23) and (22) with $i=1, j=2$ we obtain $(2 k-2) d_{2}^{2}=\mu d_{1}$. Similarly, with $i=1, j=3$, we get $2(2 k-3) d_{2}^{2}=2 \mu d_{1}$. Hence $2 k-2=2 k-3$, which is impossible.

It remains to consider $m=k=2$. Then, from (23) and (22) with $i=$ $1, j=2$, we have

$$
2 d_{2}^{2}=\mu d_{1} .
$$

Note that (17)-(20) imply that $\bar{u}_{1,1}=0, \bar{u}_{2,1}=d_{2}, \bar{u}_{1,2}=3 d_{2}, \bar{u}_{2,2}=2 d_{2}$. Hence, by (12) and (23),

$$
g(x)=g(y) g\left(y+3 d_{2}\right), \quad g\left(x+d_{1}\right)=g\left(y+d_{2}\right) g\left(y+2 d_{2}\right) .
$$

Write $g(X)=X^{\mu}+a X^{\mu-1}+b X^{\mu-2}+O\left(X^{\mu-3}\right)$. Then the first equation of (25) implies $x=y^{2}+O(y)$ in obvious notation. By computing the higher order terms we obtain

$$
g\left(x+d_{1}\right)-g(x)=\mu d_{1} x^{\mu-1}+O\left(x^{\mu-2}\right)
$$

and

$$
g\left(y+d_{2}\right) g\left(y+2 d_{2}\right)-g(y) g\left(y+3 d_{2}\right)=\left(2 \mu^{2}-4\left(\begin{array}{c}
\mu \\
2
\end{array}\right)\right) d_{2}^{2} y^{2 \mu-2}+O\left(y^{2 \mu-3}\right) .
$$


Hence, on using (25) and substituting $x=y^{2}+O(y)$,

$$
d_{1}=2 d_{2}^{2}+O(1 / y) .
$$

Together with (24) this implies $\mu=1$. Thus $g(X)=X+a$ with $a \in \mathbb{Q}$. By (25) we find that $a \in \mathbb{Z}$ and (5) follows. This completes the proof of Theorem (b).

\section{References}

[1] R. Balasubramanian and T. N. Shorey, On the equation $f(x+1) \ldots f(x+k)=$ $f(y+1) \ldots f(y+m k)$, Indag. Math. N.S. 4 (1993), 257-267.

[2] J. W. S. Cassels, Factorization of polynomials in several variables, in: Proc. 15th Scandinavian Congress, Oslo 1968, Lecture Notes in Math. 118, Springer, 1970, 1-17.

[3] N. Saradha, T. N. Shorey and R. Tijdeman, On arithmetic progressions with equal products, Acta Arith. 68 (1994), 89-100.

[4] T. N. Shorey, On a conjecture that a product of $k$ consecutive positive integers is never equal to a product of $m k$ consecutive positive integers except for $8 \cdot 9 \cdot 10=6$ ! and related questions, in: Number Theory, Séminaire de Théorie des Nombres, Paris 1992-3, S. David (ed.), London Math. Soc. Lecture Note Ser. 215, Cambridge Univ. Press, 1995, 231-244.

SCHOOL OF MATHEMATICS TATA INSTITUTE OF FUNDAMENTAL RESEARCH HOMI BHABHA ROAD

BOMBAY 400005, INDIA
MATHEMATICAL INSTITUTE LEIDEN UNIVERSITY P.O. BOX 9512 2300 RA LEIDEN, THE NETHERLANDS 